\title{
Issues on global stabilization of linear systems subject to actuator saturation *
}

\author{
Tao Yang* Anton A. Stoorvogel ${ }^{* *}$ Ali Saberi ${ }^{* * *}$ \\ * School of Electrical Engineering and Computer Science, Washington \\ State University, Pullman, WA 99164-2752 USA (e-mail: \\ tyang1@eecs.wsu.edu) \\ ** Department of Electrical Engineering, Mathematics and Computer \\ Science, University of Twente, P.O. Box 217, Enschede, The \\ Netherlands. (e-mail: A.A.Stoorvogel@utwente.nl) \\ *** School of Electrical Engineering and Computer Science, \\ Washington State University, Pullman, WA 99164-2752 USA (e-mail: \\ saberi@eecs.wsu.edu)
}

\begin{abstract}
This paper reexamines the classical issue whether linear or nonlinear static state feedback control laws are needed for globally stabilizing linear systems subject to actuator saturation.
\end{abstract}

Keywords: Systems Subject to Constraints, Nonlinear Systems, Constrained Control

\section{INTRODUCTION}

Linear systems subject to actuator saturation are ubiquitous and have been the subject of extensive study. See for instance two special issues Bernstein and Michel (1995); Saberi and Stoorvogel (1999), and references therein. Internal stabilization for this class of systems has a long history. Fuller (1969) established that a chain of integrators with order greater or equal to three cannot be globally stabilized by any saturating linear static state feedback control law with only one input channel. Sontag and Sussmann (1990) established that, global stabilization of continuous-time linear systems with bounded input can be achieved if and only if the linear system, in the absence of actuator saturation, is stabilizable and critically unstable (equivalently, asymptotically null controllable with bounded control). In general, this requires nonlinear control laws. However, for certain cases, global stabilization can be achieved by linear control laws. More precisely, the paper Tyan and Bernstein (1999) noted that systems which are asymptotically null controllable with bounded inputs can be globally stabilized by linear static state feedback control laws if all non-zero eigenvalues on the imaginary axis are semi-simple (geometric and algebraic multiplicities are equal) while zero is allowed to be an eigenvalue whose Jordan blocks can be at most of size $2 \times 2$ (which are associated with double integrators). The quoted paper Tyan and Bernstein (1999) does not give a full proof of this result. In this paper we prove this result. Moreover, our proof is constructive.

Another issue is that in the literature, there is this general belief that if there are Jordan blocks of size greater or equal to three associated to an eigenvalue in zero then

\footnotetext{
^ The work of Ali Saberi and Tao Yang is partially supported by National Science Foundation grant NSF-0901137, NAVY grants ONR KKK777SB001 and ONR KKK760SB0012.
}

one need nonlinear control laws to globally stabilize such linear systems subject to actuator saturation. This is a misconception. Such a misconception is possibly based on a misreading of the result of Fuller (1969). One should emphasize that the beautiful result of Fuller does not claim anything beyond linear static state feedback control laws for chains of integrators. In this paper we illustrate this issue by showing that a triple-integrator with multiple inputs subject to actuator saturation can be globally stabilized by linear static state feedback control laws. This is clearly a first step towards a better understanding when nonlinear static state feedback control laws are needed.

Two general open problems are still unresolved: (1) under what conditions one can utilize a linear static state feedback control law to globally stabilize a linear system subject to actuator saturation?, and (2) under what conditions one can utilize a linear dynamic state feedback control law to globally stabilize a linear system subject to actuator saturation?

\section{DESIGN LINEAR STATIC STATE FEEDBACK CONTROL LAWS FOR MIXED CASE}

In this section, we investigate when a linear static state feedback control law can globally asymptotically stabilize linear systems subject to actuator saturation. It is well known that both double-integrator and neutrally stable systems subject to actuator saturation can be globally stabilized by linear static state feedback control laws. However, the mixture of these cases is not well studied. For the mixed case, Tyan and Bernstein (1999) gave a sufficient condition that guarantees global stability of the closed-loop system by using linear static state feedback control laws, but this result is not studied from a design point of view. In contrast, we design a linear static state feedback control law to globally stabilize the mixed system subject to actuator saturation. 
We first present an algorithm which gives us a methodology for designing a linear static state feedback control law for systems consisting of double integrators, single integrators and neutrally stable dynamics subject to actuator saturation in section 2.1. Next, we prove that such a control law globally stabilizes the mixed system subject to actuator saturation via a Lyapunov argument in section 2.2. Finally, an illustrative example is given in section 2.3 .

Consider a continuous-time linear system subject to actuator saturation described by

$$
\dot{\tilde{x}}=\tilde{A} \tilde{x}+\tilde{B} \sigma(u)
$$

where $\tilde{x} \in \mathbb{R}^{n}, \tilde{u} \in \mathbb{R}^{m}$, and

$$
\sigma(u)=\left(\begin{array}{c}
\sigma_{1}\left(u_{1}\right) \\
\sigma_{1}\left(u_{2}\right) \\
\vdots \\
\sigma_{1}\left(u_{m}\right)
\end{array}\right)
$$

where $\sigma_{1}\left(u_{i}\right)$ is the standard saturation function

$$
\sigma_{1}\left(u_{i}\right)=\operatorname{sgn}\left(u_{i}\right) \min \left\{1,\left|u_{i}\right|\right\} .
$$

We assume that the pair $(\tilde{A}, \tilde{B})$ is stabilizable, and eigenvalues of $\tilde{A}$ are all located in the closed left-half complex plane (i.e. the pair $(\tilde{A}, \tilde{B})$ is asymptotically null controllable with bounded control). Furthermore, we assume that $\tilde{A}$ has eigenvalue zero with geometric multiplicity $m$ and algebraic multliplicity $m+q$ with no Jordan blocks of size larger than 2 while the remaining eigenvalues are simple purely imaginary eigenvalues. Obviously, for such systems, stabilizability of the pair $(\tilde{A}, \tilde{B})$ is equivalent to controllability of the pair $(\tilde{A}, \tilde{B})$.

\subsection{Algorithm}

The algorithm for designing a linear static state feedback control law to globally stabilize the system described in (1) is carried out in three steps:

Step 1: We can obviously find a basis transformation $\Gamma_{x}$ such that

$$
A=\Gamma_{x}^{-1} \tilde{A} \Gamma_{x}=\left(\begin{array}{ccc}
A_{d} & 0 & 0 \\
0 & A_{s} & 0 \\
0 & 0 & A_{\omega}
\end{array}\right)
$$

with

$$
A_{d}=\left(\begin{array}{ll}
0 & I \\
0 & 0
\end{array}\right)
$$

while $A_{s}=0$ and $A_{\omega}$ satisfies $A_{\omega}+A_{\omega}^{\mathrm{T}}=0$. With respect to this basis transformation we obtain:

$$
B=\Gamma_{x}^{-1} \tilde{B}=\left(\begin{array}{c}
B_{d} \\
B_{s} \\
B_{\omega}
\end{array}\right)
$$

with

$$
B_{d}=\left(\begin{array}{l}
B_{d, 1} \\
B_{d, 2}
\end{array}\right)
$$

compatible with the structure of $A_{d}$. The system in the new coordinates is given by:

$$
\dot{x}=A x+B \sigma(u) \text {. }
$$

Step 2: Design $K$ such that

$$
K=\left(\begin{array}{c}
K_{1} \\
K_{2} \\
\vdots \\
K_{m}
\end{array}\right)
$$

satisfies

$$
\begin{array}{r}
K A+B^{\mathrm{T}} \Lambda=0 \\
K B+(K B)^{\mathrm{T}}<0
\end{array}
$$

where $\Lambda$ is a diagonal matrix such that:

$$
\Lambda=\left(\begin{array}{ccc}
\Lambda_{1} & 0 & 0 \\
0 & 0 & 0 \\
0 & 0 & I
\end{array}\right)
$$

with

$$
\Lambda_{1}=\left(\begin{array}{ll}
0 & 0 \\
0 & I
\end{array}\right)
$$

compatible with the structure of $A_{d}$. The existence of such $K$ is shown in the proof of Theorem 3 .

Step 3: The linear static state feedback control law

$$
u=\tilde{K} \tilde{x}
$$

where $\tilde{K}=K \Gamma_{x}^{-1}$ globally stabilizes the system described in (1).

\subsection{Theorem}

In order to show our main theorem, we need the following lemmas. These two lemmas are very well-known and can be found in Lancaster (1969) and Khalil (1996) respectively.

Lemma 1. Given two matrices $X$ and $Y$, there exists a matrix $Z$ such that

$$
Z X=Y
$$

if and only if

$$
\operatorname{ker} X \subset \operatorname{ker} Y \text {, }
$$

where $\operatorname{ker} A$ is the null space of a matrix $A \in \mathbb{R}^{m \times n}$ defined as

$$
\operatorname{ker} A:=\left\{x \in \mathbb{R}^{n} \mid A x=0\right\} .
$$

Here, we present a special case of LaSalle's invariance principle, where $V(x)$ is positive definite, which is also known as Krasovskii Theorem.

Lemma 2. Consider the system

$$
\dot{x}=f(x)
$$

where $f: \mathbb{R}^{n} \rightarrow \mathbb{R}^{n}$.

Let $x=0$ be an equilibrium point. Let $V: \mathbb{R}^{n} \rightarrow \mathbb{R}$ be a continuously differentiable, radially unbounded, positive definite function such that $\dot{V}(x) \leq 0$ for all $x \in \mathbb{R}^{n}$. Let $\mathcal{S}=\left\{x \in \mathbb{R}^{n} \mid \dot{V}(x)=0\right\}$ and suppose that no solution can stay in $\mathcal{S}$ for all $t \geq 0$, other than the trivial solution $x(t)=0$ for all $t \geq 0$. Then, the origin is globally asymptotically stable.

Now, we show that the control law constructed above in (3) can globally stabilize the system (1).

Theorem 3. Consider a linear system as given in (1). Assume that the pair $(\tilde{A}, \tilde{B})$ is controllable. Moreover, we assume that $\tilde{A}$ has eigenvalue zero with geometric multiplicity $m$ and algebraic multliplicity $m+q$ with no Jordan blocks of size larger than 2 while the remaining eigenvalues are simple purely imaginary eigenvalues. The linear state feedback control law $u=\tilde{K} \tilde{x}$ given in (3) with appropriate gain matrix $\tilde{K}$ can globally stabilize the system (1). 
Proof. Through step 1 of the algorithm, we can transfer the system (1) into (2) as

$$
\dot{x}=A x+B \sigma(u) \text {. }
$$

The state vector has a decomposition:

$$
x=\left(\begin{array}{c}
x_{d} \\
x_{s} \\
x_{\omega}
\end{array}\right) .
$$

compatible to the decomposition of $A$. Moreover,

$$
x_{d}=\left(\begin{array}{l}
x_{d, 1} \\
x_{d, 2}
\end{array}\right) .
$$

We prove the theorem via Lyapunov argument, consider a Lyapunov candidate

$$
V(x)=\frac{1}{2} x_{\omega}^{\mathrm{T}} x_{\omega}+\frac{1}{2} x_{d, 2}^{\mathrm{T}} x_{d, 2}+\sum_{i=1}^{m} \int_{0}^{K_{i} x} \sigma_{1}(y) d y .
$$

The evaluation of $\dot{V}(x)$ along the trajectories of the closedloop system, yields,

$$
\dot{V}(x)=x_{\omega}^{\mathrm{T}} \dot{x}_{\omega}+x_{d, 2}^{\mathrm{T}} \dot{x}_{d, 2}+\sigma(K x) K \dot{x} .
$$

With some algebra, we can write the above equation in the matrix form as

$$
\dot{V}(x)=\sigma^{\mathrm{T}}(K x)\left(K A x+B^{\mathrm{T}} \Lambda x\right)+\sigma^{\mathrm{T}}(K x) K B \sigma(K x) .
$$

In order to make $\dot{V}(x)$ non-positive, it is sufficient to guarantee that the gain matrix $K$ satisfies

$$
\begin{array}{r}
K A+B^{\mathrm{T}} \Lambda=0 \\
K B+(K B)^{\mathrm{T}}<0 .
\end{array}
$$

Let us write the equation (6) in matrix equality form

$$
K(A B)=\left(-B^{\mathrm{T}} \Lambda S\right)
$$

where $S$ is any matrix satisfying $S+S^{\mathrm{T}}<0$, we get:

$$
K B+(K B)^{\mathrm{T}}=S+S^{\mathrm{T}}<0 .
$$

Now, let us show that a $K$ which satisfies the equation (7) exists. From Lemma 1, we see that to show the solvability of equation (7) is equivalent to show

$$
\left(-B^{\mathrm{T}} \Lambda S\right)\left(\begin{array}{l}
x \\
u
\end{array}\right)=0
$$

given

$$
\left(\begin{array}{ll}
A & B
\end{array}\right)\left(\begin{array}{l}
x \\
u
\end{array}\right)=0 \text {. }
$$

Since the pair $(A, B)$ is controllable, from the Hautus test Hautus (1969), we know that

$$
\operatorname{rank}(A B)=n \text {, }
$$

Moreover, from the structure of matrices $A$ and $B$, we know rank $A=n-m$ and $\operatorname{rank} B=m$. Thus

$$
\operatorname{im} A \cap \operatorname{im} B=0,
$$

where $\operatorname{im} Z$ is the range space of a matrix $Z \in \mathbb{R}^{m \times n}$ defined as

$$
\operatorname{im} Z:=\left\{Z x \mid x \in \mathbb{R}^{n}\right\} .
$$

Therefore, the equation (9), implies $A x=0$ and $B u=0$. Clearly $A x=0$ implies $x_{d, 2}=0$ and $x_{\omega}=0$ which yields $\Lambda x=0$. Hence

$$
-B^{\mathrm{T}} \Lambda x=0 .
$$

Moreover $\operatorname{rank} B=m$ while $B$ has $m$ columns yields that $B$ is injective. Therefore $B u=0$ implies $u=0$ and therefore $S u=0$. Hence (8) is satisfied and we have shown that the equation $(7)$ is solvable. Since $(A B)$ is surjective, for any given $S$, we have a unique solution $K$ for the equation (7) such that

$$
\dot{V}(x)=\sigma^{\mathrm{T}}(K x) K B \sigma(K x) \leq 0
$$

provided $S+S^{\mathrm{T}}<0$. In order to prove asymptotic stability we apply Lemma 2. Clearly our Lyapunov candidate function $V(x)$ given in (4) is continuously differentiable, radially unbounded, positive definite function.

Next, we note that the $\dot{V}(x)=0$ if and only if $K x=0$. When $K x=0$, the dynamics obviously becomes $\dot{x}=A x$. We need to show that there exists no initial condition $x(0)=x_{0} \neq 0$ such that $K x(t)=0$ for all $t>0$ while $\dot{x}(t)=A x(t)$. We have:

$$
x(t)=\left(\begin{array}{c}
x_{d, 1}(0)+t x_{d, 2}(0) \\
x_{d, 2}(0) \\
x_{s}(0) \\
x_{\omega}(t)
\end{array}\right) .
$$

Since $x_{\omega}(t)$ is only related to non-zero eigenvalues, we get from $K x(t)=0$ for all $t \geq 0$ that:

$$
K\left(\begin{array}{c}
x_{d, 2}(0) \\
0 \\
0 \\
0
\end{array}\right)=0, \quad K\left(\begin{array}{c}
x_{d, 1}(0) \\
x_{d, 2}(0) \\
x_{s}(0) \\
0
\end{array}\right)=0
$$

The first equality in (10) implies:

$$
0=K\left(\begin{array}{c}
x_{d, 2}(0) \\
0 \\
0 \\
0
\end{array}\right)=K A\left(\begin{array}{c}
0 \\
x_{d, 2}(0) \\
0 \\
0
\end{array}\right)=-B^{\mathrm{T}}\left(\begin{array}{c}
0 \\
x_{d, 2}(0) \\
0 \\
0
\end{array}\right) \text {, }
$$

which yields $B_{d, 2}^{\mathrm{T}} x_{d, 2}(0)=0$. Controllability of the pair $(A, B)$ implies that $B_{d, 2}$ must be surjective. Hence $B_{d, 2}^{\mathrm{T}}$ is injective and we obtain $x_{d, 2}(0)=0$. Next, we note that the second equality in (10) yields:

$$
\left(\begin{array}{c}
x_{d, 1}(0) \\
0 \\
x_{s}(0) \\
0
\end{array}\right)=A x+B u \text { where } x=\left(\begin{array}{c}
x_{1} \\
x_{2} \\
x_{3} \\
x_{4}
\end{array}\right)
$$

for suitably chosen $x$ and $u$ since $(A B)$ is surjective (because of controllability). Obviously, this implies that $0=B_{d, 2} u$ while $x_{4}$ satisfies:

We find:

$$
x_{4}=-A_{\omega}^{-1} B_{\omega} u .
$$

$$
\begin{aligned}
0=u^{\mathrm{T}} K\left(\begin{array}{c}
x_{d, 1}(0) \\
0 \\
x_{s}(0) \\
0
\end{array}\right) & =u^{\mathrm{T}} K[A x+B u] \\
& =-u^{\mathrm{T}} B^{\mathrm{T}} \Lambda x+u^{\mathrm{T}} S u \\
& =-u^{\mathrm{T}} B_{d, 2}^{\mathrm{T}} x_{2}-u^{\mathrm{T}} B_{\omega}^{\mathrm{T}} x_{4}+u^{\mathrm{T}} S u \\
& =u^{\mathrm{T}} B_{\omega}^{\mathrm{T}} A_{\omega}^{-1} B_{\omega} u+u^{\mathrm{T}} S u \\
& =u^{\mathrm{T}} S u
\end{aligned}
$$

where we used that $B_{d, 2} u=0$ and the fact that $A_{\omega}^{-1}$ is skew-symmetric. Since $S+S^{\mathrm{T}}<0$, we find $u=0$. But this immediately yields that $x_{s}(0)=0$. Using that $x_{s}(0)=0$ and $x_{d, 2}(0)=0$, we get from the second equality in (10) that

$$
0=K\left(\begin{array}{c}
x_{d, 1}(0) \\
0 \\
0 \\
0
\end{array}\right)=K A\left(\begin{array}{c}
0 \\
x_{d, 1}(0) \\
0 \\
0
\end{array}\right)=-B^{\mathrm{T}}\left(\begin{array}{c}
0 \\
x_{d, 1}(0) \\
0 \\
0
\end{array}\right)
$$


which yields $B_{d, 2}^{\mathrm{T}} x_{d, 1}(0)=0$. As noted before, $B_{d, 2}^{\mathrm{T}}$ is injective and therefore $x_{d, 1}(0)=0$.

It remains to show that $x_{\omega}(0)=0$. We note that

$$
K\left(\begin{array}{c}
0 \\
0 \\
0 \\
x_{\omega}(0)
\end{array}\right)=K_{\omega} x_{\omega}(0)=0,
$$

where $K_{\omega}$ is the gain matrix associated with neutrally stable dynamics. We know that $x(t)$ remains in the kernel of $K$ with $u(t)=0$. Hence:

$$
K A\left(\begin{array}{c}
0 \\
0 \\
0 \\
x_{\omega}(0)
\end{array}\right)=K_{\omega} A_{\omega} x_{\omega}(0)=0 .
$$

But since $K A=-B^{\mathrm{T}} \Lambda$ this yields:

$$
B_{\omega}^{\mathrm{T}} x_{\omega}(0)
$$

Hence if $x_{\omega}(0) \neq 0$, we have a non-trivial $A_{\omega}$-invariant subspace which is contained in $\operatorname{ker} B_{\omega}^{\mathrm{T}}$. Using the skewsymmetry of $A_{\omega}$ we find that this subspace is also $A_{\omega^{-}}^{\mathrm{T}}$ invariant. However, the existence of a non-trivial $A_{\omega}^{\mathrm{T}}$ invariant subspace contained in $\operatorname{ker} B_{\omega}^{\mathrm{T}}$ yields a contradiction with the observability of the pair $\left(B_{\omega}^{\mathrm{T}}, A_{\omega}^{\mathrm{T}}\right)$ or, equivalently a contradiction with the controllability of the pair $\left(A_{\omega}, B_{\omega}\right)$. Therefore $x_{\omega}(0)=0$.

Hence, the origin is the only solution within the subset of $\mathbb{R}^{n}$ for which $\dot{V}(x)=0$. Hence, the global asymptotic stability of the closed-loop system follows from LaSalle's Invariance Principle.

\subsection{Example}

In this section, our methodology for designing a globally stabilizing linear static state feedback control law will be illustrated by an example. Let us consider an example in form of (2), which contains two double integrators, one single integrator and neturally stable dynamics with $A$ and $B$ as follows:

$$
A=\left(\begin{array}{ccccccc}
0 & 0 & 1 & 0 & 0 & 0 & 0 \\
0 & 0 & 0 & 1 & 0 & 0 & 0 \\
0 & 0 & 0 & 0 & 0 & 0 & 0 \\
0 & 0 & 0 & 0 & 0 & 0 & 0 \\
0 & 0 & 0 & 0 & 0 & 0 & 0 \\
0 & 0 & 0 & 0 & 0 & 0 & 1 \\
0 & 0 & 0 & 0 & 0 & -1 & 0
\end{array}\right), \quad B=\left(\begin{array}{lll}
0 & 1 & 3 \\
0 & 0 & 5 \\
1 & 2 & 4 \\
0 & 1 & 6 \\
0 & 0 & 1 \\
1 & 1 & 0 \\
1 & 0 & 1
\end{array}\right) .
$$

It is easy to check that the pair $(A, B)$ is controllable. Also we notice that since $S$ is an arbitrary such that $S+S^{\mathrm{T}}<0$, the solution for the equation (7) is not unique, therefore, the linear static state feedback control laws which can globally stabilize the closed-loop system is not unique either. However, for a given $S$, we have a unique solution for the equation (7), therefore, we have a unique linear static state feedback control laws which can globally stabilize the closed-loop system.

For this example, we choose

$$
S=\left(\begin{array}{ccc}
-1 & -1 & 1 \\
1 & -3 & 1 \\
-1 & -1 & -53
\end{array}\right) \text {. }
$$

Then the unique possible globally stabilizing linear static state feedback control law is $u=K x$, where

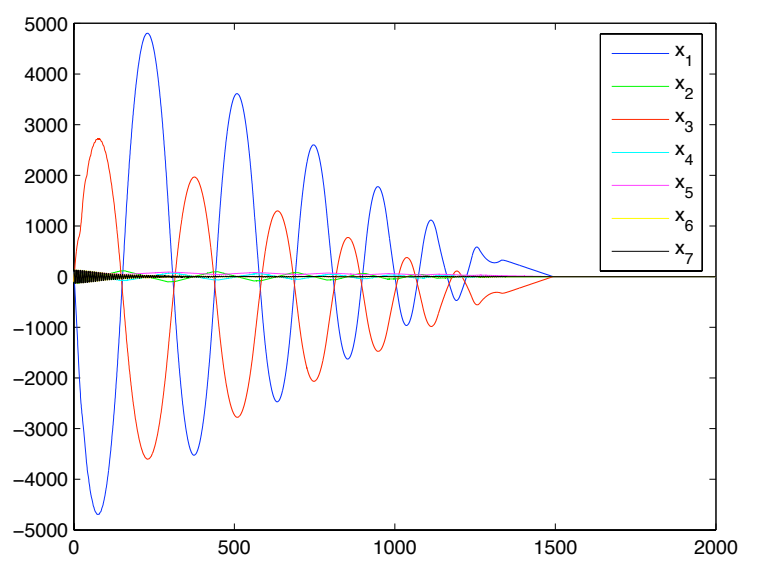

Fig. 1. Global stabilization via a linear static state feedback

$$
K=\left(\begin{array}{l}
K_{1} \\
K_{2} \\
K_{3}
\end{array}\right)
$$

with $K_{1}, K_{2}$ and $K_{3}$ given as below:

$$
\begin{aligned}
& K_{1}=\left(\begin{array}{lllllll}
-1 & 0 & -1 & 3 & -11 & -1 & 1
\end{array}\right), \\
& K_{2}=\left(\begin{array}{lllllll}
-2 & -1 & 0 & -1 & 17 & 0 & 1
\end{array}\right), \\
& \text { and } K_{3}=\left(\begin{array}{lllllll}
-4 & -6 & 0 & 4 & -35 & -1 & 0
\end{array}\right) \text {. }
\end{aligned}
$$

For the initial condition

$$
x_{0}=\left(\begin{array}{lllll}
100-100-100100100-100 & 100
\end{array}\right)^{\mathrm{T}},
$$

the dynamics are shown in Figure 1, which clearly shows that the closed-loop system is asymptotically stable.

\section{TRIPLE INTEGRATOR WITH MULTIPLE INPUTS}

In the saturation literature, it is a general belief that for a system which has an eigenvalue in zero with associated Jordan block of size greater or equal to three, there does not exist a saturating linear static state feedback control law which can globally stabilize the system. We claim that this is a misconception. More precisely, whether a saturating linear static state feedback control law exists does not only depend on the size of Jordan block. The following triple-integrator example illustrate this.

$$
\left(\begin{array}{c}
\dot{x}_{1} \\
\dot{x}_{2} \\
\dot{x}_{3}
\end{array}\right)=\left(\begin{array}{lll}
0 & 1 & 0 \\
0 & 0 & 1 \\
0 & 0 & 0
\end{array}\right)\left(\begin{array}{l}
x_{1} \\
x_{2} \\
x_{3}
\end{array}\right)+\left(\begin{array}{ll}
0 & 0 \\
0 & 1 \\
1 & 0
\end{array}\right)\left(\begin{array}{l}
\sigma_{1}\left(u_{1}\right) \\
\sigma_{1}\left(u_{2}\right)
\end{array}\right)
$$

We will prove that there exists a saturating linear static state feedback control law which can globally asymptotically stabilize the system. However, we will first present a useful lemma, see Sontag and Sussmann (1990); Sussmann and Kokotovic (1991), that we will use later:

Lemma 4. Assume that $\dot{\zeta}=f(\zeta, v)$ has a globally Lipschitz right-hand side, and that the origin is a globally asymptotically stable state for $\dot{\zeta}=f(\zeta, 0)$. Then there exists some $\lambda>0$ such that every solution of $\dot{\zeta}=f(\zeta, v)$ converges to zero, for every $v$ such that $\|v(t)\| \leq \kappa e^{-\lambda t}$.

Theorem 5. Consider a triple-integrator subject to actuator saturation, with two input channels, described by 
(11) A linear static state feedback control law can globally asymptitically stabilize the system (11).

We will present two proofs for Theorem 5. Let us present the first one based on Lemma 4.

Proof. Consider a linear static state feedback control law

$$
\begin{aligned}
& u_{1}=-\gamma x_{3} \\
& u_{2}=-\alpha x_{1}-\beta x_{2}
\end{aligned}
$$

where $\alpha>0, \beta>0$, and $\gamma>>0$. Applying this particular state feedback control law, yields the closed-loop system

$$
\begin{gathered}
\dot{x}_{1}=x_{2} \\
\dot{x}_{2}=x_{3}+\sigma_{1}\left(-\alpha x_{1}-\beta x_{2}\right) \\
\dot{x}_{3}=\sigma_{1}\left(-\gamma x_{3}\right) .
\end{gathered}
$$

The asymptotic stability of the closed-loop system follows from the fact that the poles of the linearized system are in the open left half complex plane. In order to prove global asymptotic stability of the closed-loop system, we need to show that the closed-loop system is globally attractive.

We can view the system as two subsystems, where the dynamics of subsystem 2 (equation 13) $x_{3}(t)$ is decoupled from the dynamics of subsystem $1, x_{1}(t)$ and $x_{2}(t)$, and the dynamics of subsystem $2, x_{3}(t)$ is viewed as a disturbance into subsystem 1.

We further proceed our proof by applying Lemma 4. Let us first check all the conditions of the Lemma 4.

Consider the subsystem 1 . let us define

$$
f(x)=\left(\begin{array}{c}
x_{2} \\
x_{3}+\sigma_{1}\left(-\alpha x_{1}-\beta x_{2}\right)
\end{array}\right) .
$$

Thus, we can write the dynamics of subsystem 1 as $\dot{\zeta}=$ $f(\zeta, v)$, where

$$
\zeta=\left(\begin{array}{l}
x_{1} \\
x_{2}
\end{array}\right)
$$

and $v=x_{3}$.

Clearly, the origin is globally asymptotically stable for $\dot{\zeta}=f(\zeta, 0)$, since for $v=0$, it becomes a double-integrator subject to actuator saturation with arbitrary negative linear state feedback control law, for which is well known that the closed-loop system is globally asymptotically stable. It is also easily verified that $f$ is globally Lipschitz.

In order to apply lemma 4, we know that we must guarantee $\left\|x_{3}(t)\right\| \leq \kappa e^{-\lambda t}$ for some $\lambda$ determined by the dynamics of subsystem 1 .

Obviously, we see that for big initial condition $\left\|x_{3}(0)\right\|$, i.e. the subsystem 2 given by (13) is subject to actuator saturation at the beginning, $\left\|x_{3}(t)\right\|$ decays linearly up to certain point, once it gets out of saturation region, $\left\|x_{3}(t)\right\|$ decays exponentially fast to zero, depending on $\gamma$, thus, we can design $\gamma>\lambda$, such that $\left\|x_{3}(t)\right\| \leq \kappa e^{-\lambda t}$. We automatically see that all the conditions of Lemma 4 are satisfied, therefore, every solution of the first subsystem converges to zero. Thus, the closed-loop system is globally attractive. Hence, we have proved that the closed-loop system is globally asymptotically stable.

Now, we present the second proof by constructing a Lyapunov function. This also demonstrates the fact that searching for the Lyapunov function even for simple (low order) linear system subject to actuator saturation is very complicated. Also, the Lyapunov approach guarantees stability for all $\alpha, \beta, \gamma>0$ while Lemma 4 only proves stability for $\gamma$ sufficiently large.

Proof. Let us partitioning the $\mathbb{R}^{3}$ into 4 regions. The partitions are:

$$
\begin{aligned}
& R_{1}=\left\{\left(x_{1}, x_{2}, x_{3}\right) \in \mathbb{R}^{3}\left|x_{2} x_{3}>0,\right| \gamma x_{3} \mid>1\right\}, \\
& R_{2}=\left\{\left(x_{1}, x_{2}, x_{3}\right) \in \mathbb{R}^{3}\left|x_{2} x_{3}<0,\right| \gamma x_{3} \mid>1\right\}, \\
& R_{3}=\left\{\left(x_{1}, x_{2}, x_{3}\right) \in \mathbb{R}^{3}\left|x_{2} x_{3}>0,\right| \gamma x_{3} \mid<1\right\}, \\
& \text { and } \quad R_{4}=\left\{\left(x_{1}, x_{2}, x_{3}\right) \in \mathbb{R}^{3}\left|x_{2} x_{3}<0,\right| \gamma x_{3} \mid<1\right\} .
\end{aligned}
$$

Consider a Lyapunov candidate

$$
\begin{gathered}
V(x)=\int_{0}^{\alpha x_{1}+\beta x_{2}} \sigma_{1}(y) d y+\frac{\alpha}{2} x_{2}^{2} \\
+\frac{\alpha}{\gamma} \max \left\{0, x_{2} x_{3}, x_{2} x_{3}\left|\gamma x_{3}\right|\right\}+r \max \left\{\left(\gamma x_{3}\right)^{2},\left(\gamma x_{3}\right)^{4}\right\} .
\end{gathered}
$$

We want to show that the Lyapunov candidate shown in (14) is indeed a Lyapunov function, thus, the global asymptotic stability of the closed-loop system follows. First, it is easy to see that $V(x)$ is continuous and positive definite. Also, $V(x)$ is radially unbounded.

In order to show globally asymptotically stable of the closed-loop system, we need to show that $\dot{V}(x)$ in each region is negative.

In regions $R_{1}$ and $R_{2}$, the system is described by

$$
\begin{aligned}
& \dot{x}_{1}=x_{2} \\
& \dot{x}_{2}=x_{3}+\sigma_{1}\left(-\alpha x_{1}-\beta x_{2}\right) \\
& \dot{x}_{3}=-\operatorname{sgn}\left(\gamma x_{3}\right) .
\end{aligned}
$$

For region $R_{1}$, the evaluation of $\dot{V}(x)$ along the trajectories of the closed-loop system, yields:

$$
\begin{aligned}
\dot{V}=\sigma_{1}( & \left.\alpha x_{1}+\beta x_{2}\right)\left[\alpha x_{2}+\beta\left(x_{3}-\sigma_{1}\left(\alpha x_{1}+\beta x_{2}\right)\right)\right] \\
& +\alpha x_{2}\left[x_{3}-\sigma_{1}\left(\alpha x_{1}+\beta x_{2}\right)\right] \\
& +\alpha\left[x_{3}-\sigma_{1}\left(\alpha x_{1}+\beta x_{2}\right)\right] x_{3}\left|x_{3}\right| \\
& -2 \alpha x_{2} x_{3}-4 r \gamma\left|\gamma x_{3}\right|^{3} \\
=\beta x_{3} & \sigma_{1}\left(\alpha x_{1}+\beta x_{2}\right)-\beta \sigma_{1}^{2}\left(\alpha x_{1}+\beta x_{2}\right)-\alpha x_{2} x_{3} \\
& +\alpha\left|x_{3}\right|^{3}-\alpha \sigma_{1}\left(\alpha x_{1}+\beta x_{2}\right) x_{3}\left|x_{3}\right|-4 r \gamma\left|\gamma x_{3}\right|^{3} .
\end{aligned}
$$

Since for $\left|\gamma x_{3}\right|>1$, the following hold

$$
\begin{gathered}
x_{3} \sigma\left(\alpha x_{1}+\beta x_{2}\right) \leq\left|x_{3}\right| \leq \frac{1}{\gamma}\left|\gamma x_{3}\right| \leq \frac{1}{\gamma}\left|\gamma x_{3}\right|^{3} \\
-\sigma_{1}\left(\alpha x_{1}+\beta x_{2}\right) x_{3}\left|x_{3}\right| \leq\left|x_{3}\right|^{2}=\frac{1}{\gamma^{2}}\left|\gamma x_{3}\right|^{2} \leq \frac{1}{\gamma^{2}}\left|\gamma x_{3}\right|^{3} .
\end{gathered}
$$

We get

$\dot{V} \leq-\beta \sigma_{1}^{2}\left(\alpha x_{1}+\beta x_{2}\right)-\alpha x_{2} x_{3}-\left(4 r \gamma-\frac{\alpha}{\gamma^{2}}-\frac{\alpha}{\gamma^{3}}-\frac{\beta}{\gamma}\right)\left|\gamma x_{3}\right|^{3}$.

Choosing $r$ such that:

$$
4 r \gamma^{4}>\alpha \gamma+\alpha+\beta \gamma^{2}
$$

then yields $\dot{V}<0$.

For region $R_{2}$, the evaluation of $\dot{V}_{2}$ along the trajectories of the closed-loop system, yields, 


$$
\begin{aligned}
\dot{V}= & \sigma_{1}\left(\alpha x_{1}+\beta x_{2}\right)\left[\alpha x_{2}+\beta\left(x_{3}-\sigma_{1}\left(\alpha x_{1}+\beta x_{2}\right)\right)\right] \\
& +\alpha x_{2}\left[x_{3}-\sigma_{1}\left(\alpha x_{1}+\beta x_{2}\right)\right]-4 r \gamma\left|\gamma x_{3}\right|^{3} \\
= & \beta x_{3} \sigma_{1}\left(\alpha x_{1}+\beta x_{2}\right)-\beta \sigma_{1}^{2}\left(\alpha x_{1}+\beta x_{2}\right)+\alpha x_{2} x_{3} \\
& -4 r \gamma\left|\gamma x_{3}\right|^{3} \\
\leq & -\beta \sigma_{1}^{2}\left(\alpha x_{1}+\beta x_{2}\right)+\alpha x_{2} x_{3}-\left(4 r \gamma-\frac{\beta}{\gamma}\right)\left|\gamma x_{3}\right|^{3} .
\end{aligned}
$$

Choosing $r$ such that:

$$
4 r \gamma^{2}>\beta
$$

then yields $\dot{V}<0$.

In regions $R_{3}$ and $R_{4}$, the system is described by

$$
\begin{aligned}
& \dot{x}_{1}=x_{2} \\
& \dot{x}_{2}=x_{3}+\sigma_{1}\left(\alpha x_{1}-\beta x_{2}\right) \\
& \dot{x}_{3}=-\gamma x_{3} .
\end{aligned}
$$

For region $R_{3}$, the evaluation of $\dot{V}_{3}$ along the trajectories of the closed-loop system, yields,

$$
\begin{aligned}
\dot{V}=\sigma_{1}( & \left.\alpha x_{1}+\beta x_{2}\right)\left[\alpha x_{2}+\beta\left(x_{3}-\sigma_{1}\left(\alpha x_{1}+\beta x_{2}\right)\right)\right] \\
& +\alpha x_{2}\left[x_{3}-\sigma_{1}\left(\alpha x_{1}+\beta x_{2}\right)\right] \\
& +\frac{\alpha}{\gamma}\left[x_{3}-\sigma_{1}\left(\alpha x_{1}+\beta x_{2}\right)\right] x_{3} \\
& -\alpha x_{2} x_{3}-2 r \gamma^{3} x_{3}^{2} \\
= & \left(\beta-\frac{\alpha}{\gamma}\right) x_{3} \sigma_{1}\left(\alpha x_{1}+\beta x_{2}\right)-\beta \sigma_{1}^{2}\left(\alpha x_{1}+\beta x_{2}\right) \\
& +\left(\frac{\alpha}{\gamma}-2 r \gamma^{3}\right) x_{3}^{2} .
\end{aligned}
$$

In this case, we choose $\epsilon$ small enough such that:

$$
\frac{\epsilon}{2}\left|\beta-\frac{\alpha}{\gamma}\right| \leq \frac{\beta}{2} .
$$

Next, using

we get

$$
\left|x_{3} \sigma_{1}\left(\alpha x_{1}+\beta x_{2}\right)\right| \leq \frac{\epsilon}{2} \sigma_{1}^{2}\left(\alpha x_{1}+\beta x_{2}\right)+\frac{1}{2 \epsilon} x_{3}^{2},
$$

$$
\begin{aligned}
\dot{V} \leq-\frac{\beta}{2} \sigma_{1}^{2}\left(\alpha x_{1}+\beta x_{2}\right) & \\
& +\left(\frac{1}{2 \epsilon}\left|\beta-\frac{\alpha}{\gamma}\right|+\frac{\alpha}{\gamma}-2 r \gamma^{3}\right) x_{3}^{2} .
\end{aligned}
$$

Choosing $r$ such that:

$$
2 r \gamma^{3}>\frac{1}{2 \epsilon}\left|\beta-\frac{\alpha}{\gamma}\right|+\frac{\alpha}{\gamma},
$$

then yields $\dot{V}<0$.

For region $R_{4}$, the evaluation of $\dot{V}_{4}$ along the trajectories of the closed-loop system, yields,

$$
\begin{aligned}
\dot{V}= & \sigma_{1}\left(\alpha x_{1}+\beta x_{2}\right)\left[\alpha x_{2}+\beta\left(x_{3}-\sigma_{1}\left(\alpha x_{1}+\beta x_{2}\right)\right)\right] \\
& +\alpha x_{2}\left[x_{3}-\sigma_{1}\left(\alpha x_{1}+\beta x_{2}\right)\right]-2 r \gamma^{3} x_{3}^{2} \\
= & \beta x_{3} \sigma_{1}\left(\alpha_{1}+\beta x_{2}\right)-\beta \sigma_{1}^{2}\left(\alpha x_{1}+\beta x_{2}\right) \\
& +\alpha x_{2} x_{3}-2 r \gamma^{3} x_{3}^{2} \\
\leq & \frac{\beta}{2}\left[x_{3}^{2}+\sigma_{1}^{2}\left(\alpha x_{1}+\beta x_{2}\right)\right]-\beta \sigma_{1}^{2}\left(\alpha x_{1}+\beta x_{2}\right) \\
& +\alpha x_{2} x_{3}-2 r \gamma^{3} x_{3}^{2} \\
= & -\frac{\beta}{2} \sigma_{1}^{2}\left(\alpha x_{1}+\beta x_{2}\right)+\alpha x_{2} x_{3}-\left(2 r \gamma^{3}-\frac{\beta}{2}\right) x_{3}^{2} .
\end{aligned}
$$

Choosing $r$ such that:

$$
4 r \gamma^{3}>\beta
$$

then yields $\dot{V}<0$.

Thus, choosing $r$ sufficiently large such that all inequalities (15)-(18) are satisfied, then yields $\dot{V}(x)<0$ for all four different regions. Therefore, $\dot{V}(x)$ is negative along all trajectories unequal to zero. And for the origin, from the analysis above, we see that $\dot{V}(\mathbf{0})=0$. Thus, the closedloop system is globally asymptotically stable. Hence, the triple-integrator subject to actuator saturation can be globally asymptotically stabilized via linear static state feedback control laws.

\section{CONCLUSIONS}

In this paper, we reexamine the classical issue of requiring linear or nonlinear static state feedback control laws for globally stabilizing asymptotically null controllable with bounded control systems subject to actuator saturation. We resolve here the general misconception that the size of Jordan block associated with a zero eigenvalue determines whether linear control laws or nonlinear control laws are needed for globally stabilizing a linear system subject to actuator saturation. Also, we present here constructive linear static globally stabilizing saturated state feedback control laws for linear systems mixed with double integrators, single integrators, and neutrally stable dynamics. This is our first step towards our future goals of (1) completely characterizing the class of linear systems subject to actuator saturation which can be globally stabilized by linear static state feedback control laws, and similarly (2) completely characterizing the class of linear systems subject to actuator saturation which can be globally stabilized by linear dynamic state feedback control laws.

\section{REFERENCES}

Bernstein, D. and Michel, A. (1995). Special issue on saturating actuators. Int. J. Robust $\& 3$ Nonlinear Control, $5(5), 375-540$.

Fuller, A. (1969). In-the-large stability of relay and saturating control systems with linear controller. Int. J. Contr., 10(4), 457-480.

Hautus, M. (1969). Controllability and observability conditions of linear autonomous systems. Proc. Nederl. Akad. Wetensch., Ser. A, 72(5), 443-448.

Khalil, H.K. (1996). Nonlinear systems. Prentice Hall, Upper Saddle River, 2nd edition.

Lancaster, P. (1969). Theory of matrices. Academic Press, New York.

Saberi, A. and Stoorvogel, A. (1999). Special issue on control problems with constraints. Int. J. Robust 83 Nonlinear Control, 9(10), 583-734.

Sontag, E. and Sussmann, H. (1990). Nonlinear output feedback design for linear systems with saturating controls. In Proc. 29th CDC, 3414-3416. Honolulu.

Sussmann, H. and Kokotovic, P. (1991). The peaking phenomenon and the global stabilization of nonlinear systems. IEEE Trans. Aut. Contr., 36(4), 424-440.

Tyan, F. and Bernstein, D. (1999). Global stabilization of systems containing a double integrator using a saturated linear controller. Int. J. Robust \& Nonlinear Control, 9(15), 1143-1156. 